\title{
Suppression of Temperature Fluctuations and Energy Barrier Generation by Velocity Shear
}

\author{
J. A. Boedo, ${ }^{1}$ P. W. Terry, ${ }^{2}$ D. Gray, ${ }^{1}$ R. S. Ivanov, ${ }^{3}$ R. W. Conn, ${ }^{1}$ S. Jachmich, ${ }^{4}$ G. Van Oost,${ }^{4}$ and The TEXTOR Team ${ }^{5}$ \\ ${ }^{1}$ Department of Mechanical and Aerospace Engineering, University of California, San Diego, La Jolla, California 92093 \\ ${ }^{2}$ Department of Physics, University of Wisconsin-Madison, Madison, Wisconsin 53706 \\ ${ }^{3}$ Institute for High Temperatures IVTAN, Moscow, Russian Federation, CIS \\ ${ }^{4}$ Laboratoire de Physique des Plasmas, Koiklijke Militaire School, Brussels, Belgium \\ ${ }^{5}$ Institut fuer Plasma Physik, Forschungzentrum Juelich, Juelich, Germany
}

(Received 5 October 1999)

\begin{abstract}
First measurements of temperature fluctuations in a region of high velocity shear show that absolute and normalized fluctuation levels are reduced across the shear layer, a result that is consistent with weak parallel electron thermal conduction in the electron temperature dynamics. The concomitant reduction of temperature, density, and electric field fluctuations reduces the anomalous conducted and convected heat fluxes.
\end{abstract}

PACS numbers: $52.35 . \mathrm{Ra}, 52.25 . \mathrm{Gj}, 52.55 . \mathrm{Fa}$

The presence of localized flow shear in fluids, in general, and plasmas, in particular, can reduce turbulence levels and cross-flow transport, creating a transport barrier. Such barriers develop spontaneously in a wide variety of magnetically confined plasmas, producing transitions from a low energy confinement state ( $L$ mode) to a higher energy confinement state ( $H$ mode) [1]. The $L-H$ transition in magnetically confined plasmas is accompanied by a reduction of turbulence levels [2], confirming the paradigm that microturbulence is responsible for a considerable part of the energy and particle losses [3]. Behavior similar to that of the spontaneous $L-H$ transition is obtained by applying an external radial electric field to the plasma using an electrode [4], indicating that the radial electric field and resulting $\overrightarrow{\mathrm{E}} \times \overrightarrow{\mathrm{B}}$ rotation play a crucial role. The shearing of turbulent eddies by differential rotation has been proposed as a universal mechanism to stabilize turbulence in plasmas [5] $(\overrightarrow{\mathrm{E}} \times \overrightarrow{\mathrm{B}}$ generated $)$, but it is also postulated to operate in nonionized fluids, such as two-dimensional Navier-Stokes turbulence and the stratosphere [6]. Electrode bias experiments have clarified the role of the radial electric field and its bifurcation [7] in $L-H$ transitions by correlating the externally applied electric fields with the reduction in turbulence levels [8], formation of a transport barrier, and resulting confinement improvement. The $\vec{E} \times$ $\overrightarrow{\mathrm{B}}$ velocity shear in plasmas can affect nonlinearly saturated turbulence and reduce transport by acting on both the amplitude of the fluctuations and the phase between the density and potential fluctuations [9]. The shearing rate, $\omega_{\overrightarrow{\mathrm{E}} \times \overrightarrow{\mathrm{B}}}$, must be comparable to $\Delta \omega_{D}$, the nonlinear turbulence decorrelation rate in the absence of shear. Turbulence suppression is achieved in nonlinear simulations when the rate $\omega_{\overrightarrow{\mathrm{E}} \times \overrightarrow{\mathrm{B}}}\left(\omega_{\overrightarrow{\mathrm{E}} \times \overrightarrow{\mathrm{B}}}=d V_{\overrightarrow{\mathrm{E}} \times \overrightarrow{\mathrm{B}}} / d r\right)$ is of the order of the linear growth rate $\omega_{\text {ins }}$ of the dominant mode in the plasma [10]. Turbulent decorrelation theory [9] predicts suppression of arbitrary advected fluctuations, including temperature, under $\vec{E} \times \vec{B}$ shearing. Predictions are consistent with observations of reductions in density and potential fluctuations [10] in particle transport barriers, but no direct measurements exist quantifying temperature fluctuations in a transport barrier. We report those measurements in this paper and examine the associated heat fluxes in light of recent theoretical work [9] that has indicated that if parallel thermal conduction is strong, flow shear can reduce the particle flux, but have little effect on the heat flux. Cross-field heat transport can be expressed [11], omitting species indexes ( $e$ and $i$ ), as the sum of convective and conductive, classical $(c)$ and anomalous $(\sim)$ terms:

$$
\begin{aligned}
Q_{\mathrm{tot}} & =\bar{q}_{c}+\frac{3}{2} \bar{T} \bar{\Gamma}_{r c}+\tilde{Q}_{\mathrm{cond}}+\tilde{Q}_{\mathrm{conv}} \\
& =\bar{q}_{c}+\frac{3}{2} \bar{T} \bar{\Gamma}_{r c}+\frac{3}{2} \bar{n} \frac{\left\langle\tilde{T} \tilde{E}_{\theta}\right\rangle}{B_{\phi}}+\frac{3}{2} \tilde{\Gamma}_{r} \bar{T},
\end{aligned}
$$

where $\bar{n}$ and $\bar{T}$ are the density and temperature and $\Gamma_{r}$ is the radial particle flux. The overbar denotes averaged quantities, and the brackets denote ensemble averaging. The anomalous conducted heat flux can be written in terms of the temperature fluctuations $\tilde{T}_{e}$, poloidal field fluctuations $\tilde{E}_{\theta}$, and the cross-phase angle $\alpha^{E T}$ as

$$
\begin{aligned}
\tilde{Q}_{\text {cond }} & =\frac{3}{2} \bar{n} \frac{\left\langle\tilde{T} \tilde{E}_{\theta}\right\rangle}{B_{\phi}} \\
& =\frac{3}{2} \bar{n} \frac{\left\langle\tilde{T}^{2}\right\rangle^{1 / 2}\left\langle\tilde{E}_{\theta}^{2}\right\rangle^{1 / 2}}{B_{\phi}} \frac{\left\langle\tilde{E}_{\theta} \tilde{T}\right\rangle}{\left\langle\tilde{T}^{2}\right\rangle^{1 / 2}\left\langle\tilde{E}_{\theta}^{2}\right\rangle^{1 / 2}} \\
& =\frac{3}{2} \bar{n} \frac{\left\langle\tilde{T}^{2}\right\rangle^{1 / 2}\left\langle\tilde{E}_{\theta}^{2}\right\rangle^{1 / 2}}{B_{\phi}} \gamma_{\tilde{E} \tilde{T}} \cos \alpha^{E T} .
\end{aligned}
$$

The turbulent particle flux $\tilde{\Gamma}_{r}$ depends on the rms value of the density $\left\langle\tilde{n}_{e}^{2}\right\rangle^{1 / 2}$, the coherence $\gamma_{\tilde{E} \tilde{n}}$, and the $\tilde{n}_{e}-\tilde{E}_{\theta}$ cross phase $\alpha$,

$$
\begin{aligned}
\tilde{\Gamma}_{r} & =\frac{\left\langle\tilde{E}_{\theta} \tilde{n}_{r}\right\rangle}{B_{\phi}}=\frac{\left\langle\tilde{n}_{e}^{2}\right\rangle^{1 / 2}\left\langle\tilde{E}_{\theta}^{2}\right\rangle^{1 / 2}}{B_{\phi}} \frac{\left\langle\tilde{E}_{\theta} \tilde{n}_{r}\right\rangle}{\left\langle\tilde{n}_{e}^{2}\right\rangle^{1 / 2}\left\langle\tilde{E}_{\theta}^{2}\right\rangle^{1 / 2}} \\
& =\frac{\left\langle\tilde{n}_{e}^{2}\right\rangle^{1 / 2}\left\langle\tilde{E}_{\theta}^{2}\right\rangle^{1 / 2}}{B_{\phi}} \gamma_{\tilde{E} \tilde{n}} \cos \alpha .
\end{aligned}
$$

The $T_{e}$ fluctuations have been measured in a magnetically confined plasma in the TEXTOR tokamak. The experiments were performed in 6-s-long Ohmic $(\mathrm{OH})$ 
discharges, with toroidal magnetic field $B_{t}=2.25 \mathrm{~T}$, plasma current $I_{p}=200 \mathrm{kA}$, and chord-averaged density $\bar{n}_{e 0}=1 \times 10^{13} \mathrm{~cm}^{-3}$. A radial electric field, and resulting $\overrightarrow{\mathrm{E}} \times \overrightarrow{\mathrm{B}}$ shearing, is applied by means of an electrode inserted to a radius of $41 \mathrm{~cm}$, well inside the last closed flux surface (LCFS), located at $45.5 \mathrm{~cm}$. The electrode voltage is held constant for $1.5 \mathrm{~s}$ at levels varying from 100 to $900 \mathrm{~V}$, inducing a radial electric field that increases with voltage until a bifurcation occurs [7] and the electric field reaches a maximum of $\sim 500 \mathrm{~V} / \mathrm{cm}$ between 43.5 and $44.5 \mathrm{~cm}$. The discharge density increases by a factor of 1.5 upon bifurcation and a stationary state is reached. As the voltage and shear increase, a transport barrier (identified by a steepening of the density profile) forms [8] at $\sim 44 \mathrm{~cm}, 0.5 \mathrm{~cm}$ inside where $E_{r}$ peaks. From the electric field profile [Fig. 1(a)] and correlation time of the turbulence, we find that $\omega_{\overrightarrow{\mathrm{E}} \times \overrightarrow{\mathrm{B}}}=10^{6} \mathrm{~s}^{-1}$ after the bifurcation and $\Delta \omega_{D}=5 \times 10^{5} \mathrm{~s}^{-1}$; thus, turbulence should be well stabilized. The temperature inside the barrier [Fig. 1(b)] is reduced by almost a factor of 2 due to the intense radiation produced by carbon originated at the electrode, reaching $70 \%$ of the total input power. Conditions with no, low, and high shear are labeled $O H$ or $L$ (for Ohmic conditions with low confinement), $L-H$ (transition between low and high confinement), and $H$ (high confinement) throughout this paper. Turbulence
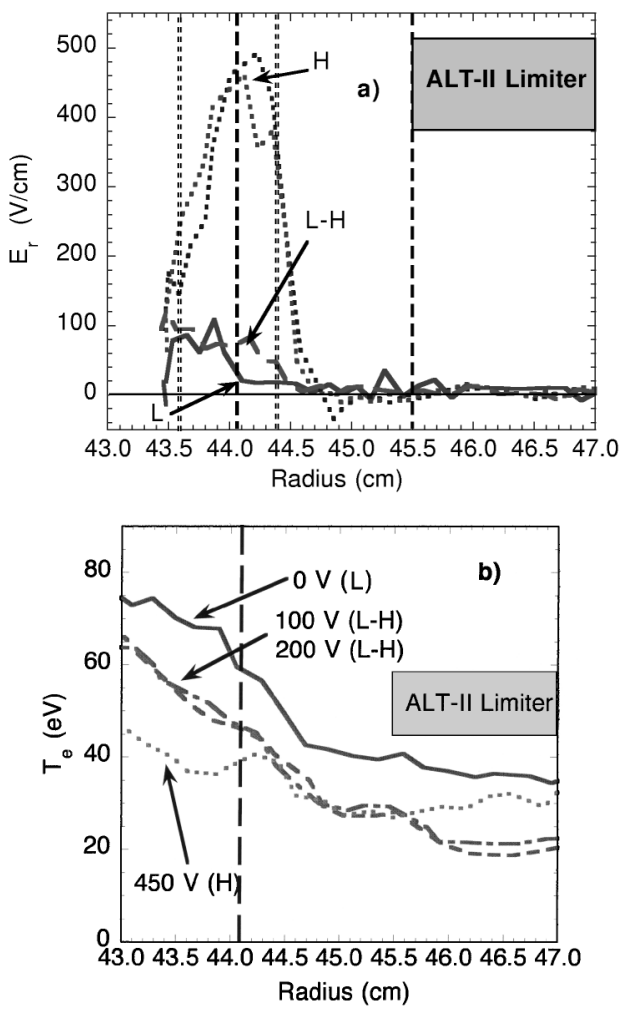

FIG. 1. Electric field (a) and temperature profiles (b) for high shear $(H)$ and $L$ discharges. The long-dashed and dotted lines mark the maximum of the radial electric field and its derivative, respectively. data were obtained with a fast reciprocating probe array featuring five $1.5 \mathrm{~mm}$ by $1.5 \mathrm{~mm}$ tips for simultaneous measurements of fluctuating floating potentials, poloidal and radial electric fields, and local plasma density. The data were digitized at 10 bit and $1 \mathrm{MHz}$. The power spectra of the density, potential, and temperature fluctuations are significant only up to $200 \mathrm{kHz}$. The temperature fluctuations are measured using the harmonics technique [12].

The main result of this work is that the temperature fluctuations are reduced by a factor of $1.5-2$ in the weaker shear conditions $(100 \mathrm{~V}, 200 \mathrm{~V})$ and by a factor of 2-3 after the bifurcation to $H$ mode $(450 \mathrm{~V})$ as shown in Fig. 2(b). Normalized temperature fluctuations [Fig. 2(a)], which are similar for weak and strong shear conditions are reduced by a factor of $\sim 2$. The experimental results can be understood by considering a simple model for the response of electron temperature fluctuations to the potential. The temperature fluctuation $\tilde{T}$, under advection of the mean temperature gradient by the $\overrightarrow{\mathrm{E}} \times \overrightarrow{\mathrm{B}}$ velocity, is governed by $\left(\partial / \partial t+V_{0} r^{-1} \partial / \partial \theta+B^{-1} \nabla \phi \times\right.$ b $\left.\cdot \nabla-\chi_{\|} \nabla_{\|}^{2}\right) \tilde{T}=B^{-1} r^{-1} \partial \phi / \partial \theta d \bar{T} / d r$, where $\chi_{\|}$is the parallel thermal conductivity, $V_{0}$ is the poloidal $\overrightarrow{\mathrm{E}} \times \overrightarrow{\mathrm{B}}$ shear flow, $\phi$ is the fluctuating electrostatic potential, and $\mathbf{b}$ is the unit vector in the direction of the magnetic field. $V_{0}$ is written as $\Delta x V_{0}^{\prime}$, where $\Delta x$ is the distance from the rational surface. If $\tilde{T}$ is governed by a collective excitation whose linear growth rate is $\omega_{\text {ins }}$ and radial mode width is $W$, the linear temperature response becomes

$$
\tilde{T}=\frac{B^{-1} \frac{\partial \phi}{r \partial \theta} \frac{d \bar{T}}{d r}}{\left(\omega_{\text {ins }}+i V_{0}^{\prime} W m / r-\chi \| W^{2} m^{2} / R^{2} q^{2} L_{q}^{2}+\Delta \omega_{D}\right)},
$$
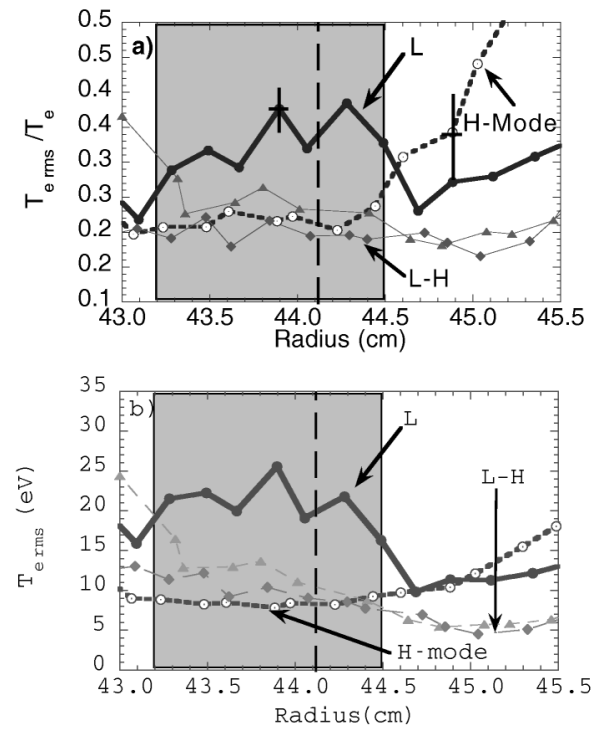

FIG. 2. Profiles of normalized (a) and absolute (b) $\tilde{T}_{e}$ for $L$, high-shear $(H$ at $450 \mathrm{~V})$, and low shear $(L-H$ at 100 and $200 \mathrm{~V})$ conditions. The shaded region marks the shear layer. 
where $m$ is the poloidal mode number, $L_{q}^{-1}=q^{-1} d q / d r$ is the scale length of the safety factor $q$, and $R$ is the major radius. The second through fourth terms of the denominator are the $\overrightarrow{\mathrm{E}} \times \overrightarrow{\mathrm{B}}$ shearing rate, the rate of parallel thermal conduction, and the nonlinear decorrelation rate, respectively. In a situation where the parallel thermal conductivity term is significantly larger than the growth rate, the nonlinear decorrelation rate, or the maximum value of the flow shear term, increases of flow shear lead to little change in temperature fluctuations because parallel thermal conduction dominates the response. On the other hand, suppression of $\tilde{T}$ should be observed if the shearing rate becomes larger than any of the other rates in the response. The terms in Eq. (4) can be estimated by considering resistive pressure gradient driven turbulence, which is an appropriate edge model for these plasmas and has been studied extensively in the context of shear-induced transport reduction. The growth rate and mode width are given by $\omega_{\text {ins }}=\left[\kappa(m / r) L_{s} \eta^{1 / 2} P_{0}^{\prime} \rho_{m}^{-1 / 2} B^{-1}\right]^{2 / 3}$ and $W=\left[L_{s}^{4} \rho_{m} \eta^{2} \kappa P_{0}^{\prime}(m / r)^{-2} B^{-4}\right]^{1 / 6}$, where $\kappa$ is the magnetic field curvature, $L_{s}=R q L_{q} r^{-1}$ is the magnetic shear scale length, $\eta$ is the resistivity, $P_{0}^{\prime}$ is the mean pressure gradient, and $\rho_{m}$ is the mass density. For the edge of the TEXTOR tokamak, the poloidal wave number, $m / r$, is $1-2 \mathrm{~cm}^{-1}, R=2.2 \mathrm{~m}, T_{0}=50 \mathrm{eV}$, $n=2.5 \times 10^{12} \mathrm{~cm}^{-3}, \quad B=1.8 \mathrm{~T}, \quad P_{0}^{\prime} / n T=1 \mathrm{~cm}^{-1}$, and $L_{s} \cong 2 R=4.4 \mathrm{~m}$. For $m / r=1 \mathrm{~cm}^{-1}$, the growth
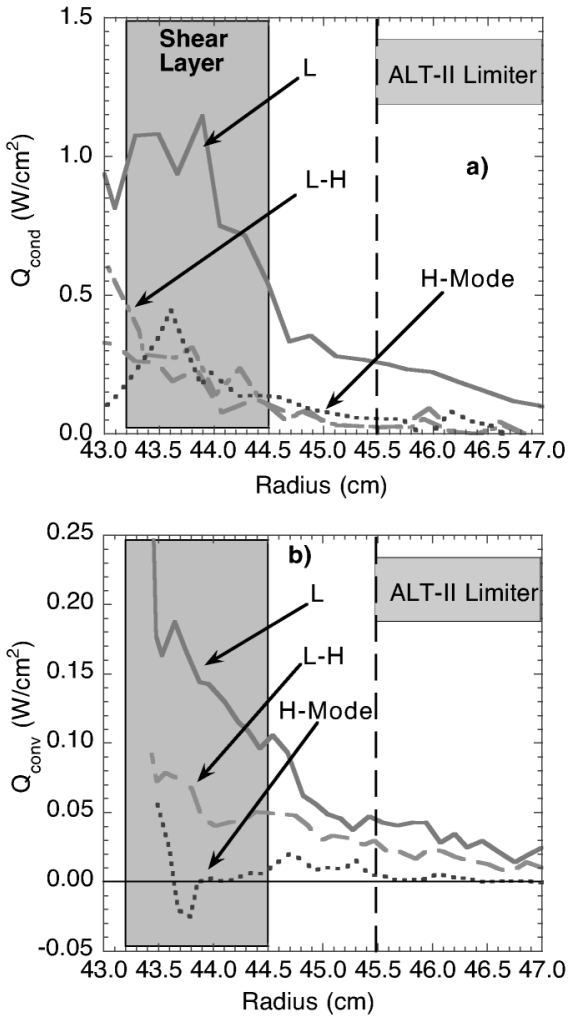

FIG. 3. Turbulent conducted (a) and convected (b) radial heat fluxes. rate is $10^{5} \mathrm{~s}^{-1}$, and the parallel thermal conduction is $5 \times 10^{4} \mathrm{~s}^{-1}$. From measurements, $\Delta \omega_{D}=5 \times 10^{5} \mathrm{~s}^{-1}$. All of these are less than $\omega_{\overrightarrow{\mathrm{E}} \times \overrightarrow{\mathrm{B}}}=V_{0}^{\prime} W m / r=10^{6} \mathrm{~s}^{-1}$, indicating that temperature fluctuations should be reduced, as observed.

The anomalous conducted electron heat profile, and its change with shear, can be calculated from Eq. (2) by using the measured values of $\tilde{T}_{e}, \tilde{E}_{\theta}, \bar{n}_{e}$, and assuming a strong $\tilde{T}_{e}-\tilde{E}_{\theta}$ correlation (i.e., $\gamma_{\tilde{E}} \tilde{T} \cos \alpha^{E T}=1$ ). The calculated flux, shown in Fig. 3(a), is an upper limit for sheared plasmas and accurate for the $O H$ conditions since a strong $\tilde{T}_{e}-\tilde{E}_{\theta}$ correlation has been documented for nonsheared plasmas in a variety of tokamaks $[13,14]$. Even the upper limit shows a factor of 4 reduction in the conducted heat flux for strong $\overrightarrow{\mathrm{E}} \times \overrightarrow{\mathrm{B}}$ shear [Fig. 3(a)], a result from reductions in $\tilde{T}_{e}$ by a factor of $2-3$ and $\tilde{E}_{\theta}$ by a factor of 3 across the shear layer. Note that the assumed cross phase should be correct for $O H$ conditions and that the value of $\gamma_{\tilde{E}} \tilde{T} \cos \alpha^{E T}$ in $H$ mode could be reduced further only by velocity shear. The anomalous convected electron heat flux [Fig. 3(b)] also decreases in the shear layer as a result [Eq. (3)] of reductions in $\tilde{E}_{\theta}$ [Fig. 4(b)] by a factor of 3 and in $\gamma_{\tilde{E} \tilde{n}} \cos \alpha\left(\gamma_{\tilde{E} \tilde{n}}\right.$ remains at $\left.\sim 0.6\right)$, which becomes negative at $43.7 \mathrm{~cm}$ [Fig. 4(c)], and causes a change in flux direction. The conduction term is larger

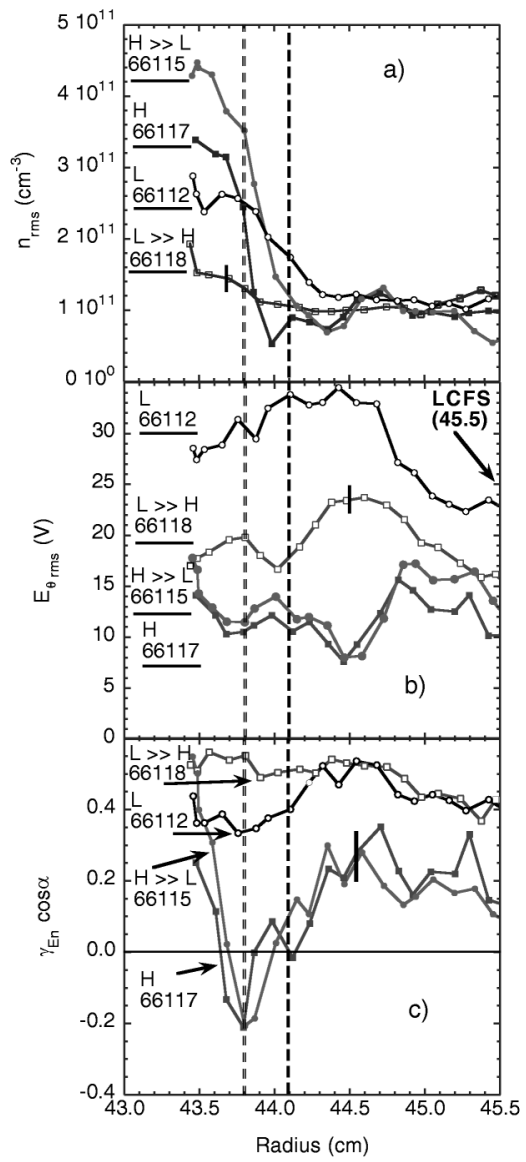

FIG. 4. Radial profiles of (a) $\tilde{n}$, (b) $\tilde{E}_{\theta}$, and (c) $\gamma_{\tilde{E} \tilde{n}} \cos \alpha$. 
TABLE I. Power balance in kW.

\begin{tabular}{ccrcccccc}
\hline \hline Mode & $P_{\text {in }}$ & $P_{\text {rad }}$ & $P_{\text {in }}-P_{\text {rad }}$ & $P_{\text {conv }}^{H_{\alpha}}$ & $\tilde{P}_{\text {conv }}^{e}$ & $\tilde{P}_{\text {cond }}^{e}$ & $\tilde{P}_{T}^{e}$ & $\tilde{P}_{T}^{i+e}$ \\
\hline OH & 250 & 87 & 163 & 41 & 17 & 80 & 97 & 194 \\
$H$ & 281 & 187 & 94 & 23 & 0.22 & 16 & 16.22 & 32.5 \\
\hline
\end{tabular}

than the convection term by a factor of $7-10$ in $O H$ conditions and, if quenched, becomes an energy barrier generator. Note that the change in $\cos \alpha$ is dependent on the sign of $V_{0}^{\prime}$ instead of the predicted [15] $V_{0}^{\prime 2}$. An increase in $\tilde{n}$ $H$-mode levels over $L$-mode levels [Fig. 4(a)] occurs when $\nabla E_{r}>0$ and $\nabla^{2} E_{r}>0$, and a reduction where $\nabla E_{r}>0$ and $\nabla^{2} E_{r}<0$ and where $\nabla E_{r}<0$ and $\nabla^{2} E_{r}<0$, showing that the density fluctuations are sensitive to higher derivatives of the velocity. The fluctuation ordering in the edge is $\tilde{T}_{e} / T_{e} \approx 0.35>\tilde{\phi} / k T_{e} \approx \tilde{n} / n \approx 0.25$ for these low-density $O H$ plasmas.

The power balance for these discharges is summarized in Table I. The difference between the input and radiated powers, $P_{\text {in }}$ and $P_{\text {rad }}( \pm 7 \%)$, is convected and conducted across the LCFS. The total power $\tilde{P}_{T}^{e}$ carried across the LCFS by the sum of turbulent electron convection $\tilde{P}_{\text {conv }}^{e}$ and conduction $\tilde{P}_{\text {cond }}^{e}$, if assumed uniform over the cross section, can account for $\sim 60 \%$ and $\sim 18 \%$ of the total in $O H$ and $H$ plasmas, respectively. If an equal ion contribution is assumed and added [13], then $\tilde{P}_{T}^{i+e} \sim 120 \%(O H)$ and $\sim 36 \%(H)$ of the total power. Therefore, anomalous transport accounts for the power balance in $\mathrm{OH}$, but not in $H$, conditions. Insight is obtained by considering the power convected by the total particle flux inferred from $H_{\alpha}, P_{\text {conv }}^{H_{\alpha}}$, which compares well to $\tilde{P}_{\text {conv }}^{e+i}$, in $O H$ conditions but is much higher in $H$ conditions. Once the dominating turbulent particle flux is quenched, other sources of transport that are significant $(\sim 40 \%)$, but unknown, become apparent. If transport reductions were limited solely to the particle flux in TEXTOR (i.e., no reduction in conducted heat flux), the total heat flux would experience only a slight decrease from 194 to $160 \mathrm{~kW}$ and mimic results where a particle barrier, and not an energy barrier, was observed $[2,16]$.

In summary, first measurements of the suppression of $T_{e}$ fluctuations in a strongly sheared velocity field are presented, as predicted by theory if parallel thermal conduction is not dominant. Reductions in poloidal electric field, temperature, and density fluctuations across the shear layer lead to a reduction of the anomalous conducted and convected heat fluxes resulting in an energy transport barrier that is measured directly. Anomalous conduction is $\sim 10$ times greater than anomalous convection in $\mathrm{OH}$ conditions, and the latter shows a dependence on higher derivatives of the shear velocity.

The authors acknowledge discussions with S. Krasheninnikov and Professor Vandenplas. This work has been supported by DOE Contract No. DEFG03-85ER51069, by the ERM/KMS Brussels, and by the IPP, FZ Juelich. The authors thank Professor Vandenplas, Professor Wolf, and especially L. Russo for their support.

[1] The ASDEX Team, Nucl. Fusion 29, 1959 (1989).

[2] G. R. Tynan et al., Phys. Plasmas 1, 3301 (1994).

[3] W. Rowan et al., Nucl. Fusion 27, 1105 (1987).

[4] R. Taylor et al., Phys. Rev. Lett. 63, 2365 (1989).

[5] H. Biglari, P. H. Diamond, and P. W. Terry, Phys. Fluids B 2, 1 (1990).

[6] P. W. Terry, Rev. Mod. Phys. (to be published).

[7] J. Cornelis, R. Sporken, G. VanOost, and R. Weynants, Nucl. Fusion 34, 171 (1994).

[8] G. R. Tynan et al., J. Nucl. Mater. 196-198, 770 (1992).

[9] A. S. Ware, P. W. Terry, B. A. Carreras, and P. H. Diamond, Phys. Plasmas 5, 173 (1998).

[10] K. H. Burrell, Phys. Plasmas 4, 1499 (1997).

[11] D. W. Ross, Comments Plasma Phys. Control. Fusion 12, 155 (1989).

[12] J. Boedo et al., Rev. Sci. Instrum. 70, 2997 (1999).

[13] C. Ritz et al., Phys. Rev. Lett. 62, 1844 (1989).

[14] H. Lin, R. D. Bengtson, and C. P. Ritz, Phys. Fluids 1, 2027 (1989).

[15] A. S. Ware, P. Terry, P. Diamond, and B. Carreras, Plasma Phys. Control. Fusion 38, 1343 (1990).

[16] M. R. Stoneking, N. E. Lanier, S. C. Prager, J. S. Sarff, and D. Sinitsys, Phys. Plasmas 4, 1632 (1997). 